\title{
AdS Duals of Matrix Strings
}

\author{
Jose F. Morales ${ }^{1,2}$ and Henning Samtleben ${ }^{1}$ \\ ${ }^{1}$ ITP and Spinoza Institute, Utrecht, The Netherlands \\ ${ }^{2}$ LNF-INFN, Frascati, Rome, Italy \\ morales@phys.uu.nl, h.samtleben@phys.uu.nl
}

\begin{abstract}
We review recent work on the holographic duals of type II and heterotic matrix string theories described by warped $\mathrm{AdS}_{3}$ supergravities. In particular, we compute the spectra of Kaluza-Klein primaries for type I, II supergravities on warped $\mathrm{AdS}_{3} \times S^{7}$ and match them with the primary operators in the dual two-dimensional gauge theories. The presence of non-trivial warp factors and dilaton profiles requires a modification of the familiar dictionary between masses and "scaling" dimensions of fields and operators. We present these modifications for the general case of domain wall/QFT correspondences between supergravities on warped $\mathrm{AdS}_{d+1} \times S^{q}$ geometries and super Yang-Mills theories with 16 supercharges.
\end{abstract}

Proceedings of the RTN workshop "The quantum structure of spacetime and the geometric nature of fundamental interactions”, Leuven, September 2002

\section{Introduction}

The AdS/CFT correspondence which in its original form [1] relates M/string theory on anti-de Sitter spaces to boundary conformal field theories has been rapidly extended to account for more general M/string backgrounds and non-conformal field theories. In [2], a proposal was put forward relating string theory on near horizon Dp-brane geometries to $d=p+1$ dimensional super Yang-Mills (SYM) theories with sixteen supercharges. Despite the earlier encouraging results of [3] and the obvious interest in these so called domain wall/QFT dualities where holography is at work in the presence of non-trivial warped geometries and dilaton profiles, these nonconformal settings of the correspondence are still far from being understood. In [П], we started a systematic study of this physics. Here we review some of the the main ideas and results.

We focus on the case $d=2$ which is a particularly rich setting for a domain wall/QFT correspondence. It relates string theory on certain warped $\mathrm{AdS}_{3} \times S^{7}$ backgrounds to twodimensional fundamental string or gauge theories, depending on whether we study systems of fundamental or D-strings. Fundamental string solutions are common to all five ten-dimensional string theories. The study of the domain wall/QFT correspondence in this case is particularly interesting since the string background is free of RR fields. The case of D-strings is specific 
to type IIB and type I theory. The dual gauge theories in these cases correspond to the matrix string models [5], 6] proposed as non-perturbative definitions of type IIA and heterotic string theories. The correspondences under study here in principle provide a supergravity description of this physics (see [7] for early discussions on these ideas).

We refer the reader to [ 4 ] for more detailed discussions of the results reported here and a complete list of references.

\section{Warped $\mathrm{AdS}_{d+1} \times S^{q}$ geometries}

Let us start by extending the familiar dictionary between masses and scaling dimensions on AdS spaces to domain wall/QFT correspondences involving warped $\mathrm{AdS}_{d+1} \times S^{q}$ geometries, applying the ideas of [8]. More specifically, we consider spaces described by

$$
d \hat{s}^{2}=z^{\omega} d s^{2}=z^{\omega}\left[\frac{\ell^{2}}{z^{2}} d x^{\mu} d x_{\mu}+\widetilde{\ell^{2}} d \Omega_{q}\right]
$$

which typically arise as near horizon geometries of p-brane solutions of low energy supergravities. Greek letters $\mu=0,1, \ldots, d$, here refer to components along $\operatorname{AdS}_{d+1}$ with $x^{d} \equiv z$, while Arabic indices $m=d+1, \ldots, D-1$ run over the $S^{q}$ sphere with metric element $d \Omega_{q}$. For definiteness, let us consider Dp-branes, such that $D=10, d=p+1, q=9-d$, and (for $d \neq 6$ )

$$
\omega=-\frac{(d-4)^{2}}{4(6-d)}, \quad \widetilde{\ell}^{2}=\frac{\ell^{2}}{4}(6-d)^{2}, \quad z=\frac{2 \sqrt{c_{d} g_{\mathrm{YM}}^{2} N}}{6-d} r^{\frac{d-6}{2}},
$$

with a constant $c_{d}$, and $r$ denoting the distance from the brane source. In addition, the supergravity solutions involve a non-trivial flux for a rank $d+1$ form and (unless $d=4$ ) a running dilaton. The Yang-Mills coupling constant $g_{\mathrm{YM}}$ carries dimension of $[L]^{d-4}$. Perturbation theory is better organized in terms the dimensionless 't Hooft parameter $\lambda_{\text {eff }} \equiv c_{d} g_{\mathrm{YM}}^{2} N\left(\frac{r}{\alpha^{\prime}}\right)^{d-4}$. The gauge theory is weakly coupled for $\lambda_{\text {eff }} \ll 1$. The other two relevant parameters are the AdS radius $\ell^{2} z^{\omega} \sim \sqrt{N} \lambda_{\text {eff }}^{\frac{d-4}{4}}$ and the string coupling constant $e^{\phi} \sim \lambda_{\text {eff }}^{\frac{8-d}{4}} / N$ [2]. As usual, genus expansion corresponds to an expansion in $\frac{1}{N}$. We will always work in the limit of large $N$ with $\lambda_{\text {eff }}$ kept fixed where both supergravity and string perturbation can be trusted.

The equation of motion of a massless ten-dimensional scalar field moving freely on (2.1) can be written as

$$
\left(\square \text { AdS }+\frac{4 \omega}{\ell^{2}} \partial_{z}-m^{2}\right) \phi=0
$$

where $\square_{\text {AdS }}$ denotes the pure AdS d'Alembertian and the effect of the warped geometry shows up in the $\omega$-dependent shift of the differential operator in (2.3). The mass parameter $m$ is defined by the eigenvalue equation:

$$
\square_{S^{9-d}} \phi=-m^{2} \phi
$$


and characterizes the harmonic mode of the scalar field $\phi$ along the sphere $S^{9-d}$. After a Fourier transform in the $d$-dimensional space spanned by $\mathbf{x}=\left(x^{0}, \ldots, x^{d-1}\right)$, the scalar equation (2.3) reduces to a second order differential equation for $\phi_{p}(z)$ of the kind

$$
\left(z^{2} \partial_{z}^{2}+(1-2 a) z \partial_{z}+p^{2} z^{2}+a^{2}-\delta^{2}\right) \phi_{p}(z),
$$

with momentum $p$, and parameters

$$
a=\frac{d}{2}-2 \omega, \quad \delta=\sqrt{a^{2}+m^{2} \ell^{2}} .
$$

Its general solution can be expressed in terms of Bessel functions $J_{\delta}, Y_{\delta}$, as

$$
\phi_{p}(z)=z^{a}\left[d_{1} J_{\delta}(p z)+d_{2} Y_{\delta}(p z)\right] \approx z^{E_{0}-2 \delta}\left(\phi_{\mathrm{def}}+\ldots\right)+z^{E_{0}}\left(\phi_{\mathrm{vev}}+\ldots\right)
$$

where dots stands for subleading terms in the expansion near the boundary $z \sim 0$. The quantities $\phi_{\mathrm{def}}, \phi_{\mathrm{vev}}$ encode the boundary conditions and represent as usual deformations and non-trivial vev's in the dual gauge theory. Finally $E_{0}$, to which we will refer as the "energy", is given by

$$
E_{0}=a+\delta=a+\sqrt{a^{2}+m^{2} \ell^{2}},
$$

In the conformal case $d=4$, the energy $E_{0}$ is identified with the conformal dimension of the dual operator and governs two-point correlation functions in the gauge theory side. This result generalizes straightforwardly to our case. By plugging the solution (2.6) in the equation of motion of the scalar field in the warped geometry and following the standard holographic prescription [9] for extracting the two-point function of scalar fields one finds [\#]

$$
\langle\mathcal{O}(\mathbf{x}) \mathcal{O}(\mathbf{y})\rangle \sim|\mathbf{x}-\mathbf{y}|^{-2\left(E_{0}-a+\frac{d}{2}\right)} .
$$

The entire information about the warped geometry is contained in the parameters $E_{0}, a$. In particular, the mass bound at which the argument of the square root becomes negative is shifted in the warped geometry with respect to its pure AdS cousin. A rather non-trivial consistency check of the whole picture follows from the need of certain conspiracies between masses and warp factors entering $\delta$ in (2.7) in order to produce rational numbers as output of the square root. We will verify this by explicit calculations of the spectrum of harmonics in warped $\mathrm{AdS}_{3} \times S^{7}$.

\section{$3 \quad$ Type II supergravities on warped $\mathrm{AdS}_{3} \times S^{7}$}

The spectrum of primaries in type II supergravities on near horizon D-string geometries is found by expanding the equations of motion to linear order in the fluctuations around the corresponding warped $\mathrm{AdS}_{3} \times S^{7}$ vacuum. Ten-dimensional fluctuations are written in terms of $S^{7}$-sphere harmonics $Y_{\mathbf{m}}^{\ell}(y)$ as

$$
\Phi_{\mu \mathbf{m}}=\sum_{\ell} \phi_{\mu}^{\ell}(x) Y_{\mathbf{m}}^{\ell}(y)
$$

with collective indices $\mu, \mathbf{m}$ carrying the $S O(3) \times S O(7)$ Lorentz representation of the given field. The sum over $\ell$ runs over a set of allowed representations of the $S O(8)$ isometry group. 


\begin{tabular}{||l|l|l|l|l||}
\hline \hline$(n+2000)$ & $(n+1010)$ & $(n 100)$ & $(n 001)$ & $(n 000)$ \\
$(n+1010)$ & $(n 100)+(n 020)$ & $(n 001)+(n-1110)$ & $(n 000)+(n-1011)$ & $(n-1010)$ \\
$(n 100)$ & $(n 001)+(n-1110)$ & $(n 000)+(n-1011)+(n-2200)$ & $(n-1010)+(n-2101)$ & $(n-2100)$ \\
$(n 001)$ & $(n 000)+(n-1011)$ & $(n-1010)+(n-2101)$ & $(n-2100)+(n-2002)$ & $(n-2001)$ \\
$(n 000)$ & $(n-1010)$ & $(n-2100)$ & $(n-2001)$ & $(n-2000)$ \\
\hline \hline
\end{tabular}

Table 1: Spectrum of IIA supergravity on $S^{7}$, the multiplet (n000) IIA.

\begin{tabular}{||l|l|l|l|l||}
\hline \hline$(n+2000)$ & $(n+1001)$ & $(n 100)$ & $(n 010)$ & $(n 000)$ \\
$(n+1010)$ & $(n+1000)+(n 011)$ & $(n 001)+(n-1110)$ & $(n-1100)+(n-1020)$ & $(n-1010)$ \\
$(n 100)$ & $(n 010)+(n-1101)$ & $(n 000)+(n-1011)+(n-2200)$ & $(n-1001)+(n-2110)$ & $(n-2100)$ \\
$(n 001)$ & $(n-1100)+(n-1002)$ & $(n-1010)+(n-2101)$ & $(n-1000)+(n-2011)$ & $(n-2001)$ \\
$(n 000)$ & $(n-1001)$ & $(n-2100)$ & $(n-2010)$ & $(n-2000)$ \\
\hline \hline
\end{tabular}

Table 2: Spectrum of IIB supergravity on $S^{7}$, the multiplet $(\mathbf{n 0 0 0})_{\text {IIB }}$.

In general, the spectrum of $S O(q+1)$ representations appearing in the Kaluza-Klein reduction of a D-dimensional supergravity on the sphere $S^{q}$ is essentially determined by group theory [10] (see [11] for recent applications). The harmonic expansion of a field transforming in the $\mathcal{R}_{S O(q)}$ representation of the Lorentz group of $S^{q}$ comprises all the representations $\mathcal{R}_{S O(q+1)}$ of the isometry group $S O(q+1)$ that contain $\mathcal{R}_{S O(q)}$ in their decomposition.

The on-shell field content of ten-dimensional type II supergravities $\left(\mathbf{8}_{\mathbf{v}}+\mathbf{8}_{\mathrm{s}}\right) \times\left(\mathbf{8}_{\mathbf{v}}+\mathbf{8}_{\mathrm{s}, \mathrm{c}}\right)$ decomposes under the $S O(7)$ Lorentz group of $S^{7}$ as

$$
\mathcal{R}_{S O(7)}^{\mathrm{II}}=3 \cdot \mathbf{1}+3 \cdot \mathbf{7}+4 \cdot \mathbf{8}+2 \cdot \mathbf{2 1}+\mathbf{2 7}+\mathbf{3 5}+2 \cdot \mathbf{4 8}
$$

Every representation in (3.2) gives rise to a tower of harmonics of those $S O(8)$ representations in which it is contained upon decomposition under $S O(7)$ (see table 1 of [ 4 ). Collecting the various contributions coming from (3.2) and grouping them into supermultiplets of the superalgebra of background isometries [ $\$$ ] we arrive at the spectrum of $S O(8)$ representations given by

$$
\begin{aligned}
& \mathcal{H}_{\mathrm{IIA}}=\sum_{n=0}^{\infty}(\mathbf{n} 000)_{\mathrm{IIA}} \equiv \sum_{n=0}^{\infty}\left(\mathbf{8}_{\mathbf{v}}-\mathbf{8}_{\mathbf{s}}\right)\left(\mathbf{8}_{\mathbf{v}}-\mathbf{8}_{\mathbf{s}}\right)(n 000), \\
& \mathcal{H}_{\mathrm{IIB}}=\sum_{n=0}^{\infty}(\mathbf{n} 000)_{\mathrm{IIB}} \equiv \sum_{n=0}^{\infty}\left(\mathbf{8}_{\mathbf{v}}-\mathbf{8}_{\mathbf{s}}\right)\left(\mathbf{8}_{\mathbf{v}}-\mathbf{8}_{\mathbf{c}}\right)(n 000),
\end{aligned}
$$

for type IIA and IIB, respectively. The supermultiplets (n000) $)_{\text {IIA,IIB }}$ are displayed in tables 1, 2 (the omission of any representation with negative Dynkin labels is always understood). The quantum numbers $\ell_{0}, \bar{\ell}_{0}$, defined in terms of the energy $E_{0}=\ell_{0}+\bar{\ell}_{0}$ and spin $s=\ell_{0}-\bar{\ell}_{0}$ may be extracted from these tables as follows: The state in the upper left corner has $\left(\ell_{0}, \bar{\ell}_{0}\right)=$ $\left(\frac{1}{4}(n+2), \frac{1}{4}(n+2)\right)$. The 8 unbroken supersymmetry generators act vertically in this table, increasing the value of $\bar{\ell}_{0}$ from top to bottom by $\frac{1}{2}$ per row. The value of $\ell_{0}$ is increased from left to right by $\frac{1}{2}$ per column which may be thought of as the action of the broken supersymmetry 


\begin{tabular}{||l|l|l|l||}
\hline \hline$(n+2000)$ & $(n 100)$ & $(n 000)$ & $n_{v} \cdot(n+1000)$ \\
$(n+1010)$ & $(n 001)+(n-1110)$ & $(n-1010)$ & $n_{v} \cdot(n 010)$ \\
$(n 100)$ & $(n 000)+(n-1011)+(n-2200)$ & $(n-2100)$ & $n_{v} \cdot(n-1100)$ \\
$(n 001)$ & $(n-1010)+(n-2101)$ & $(n-2001)$ & $n_{v} \cdot(n-1001)$ \\
$(n 000)$ & $(n-2100)$ & $(n-2000)$ & $n_{v} \cdot(n-1000)$ \\
\hline \hline
\end{tabular}

Table 3: Spectrum of I supergravity on $S^{7}$.

generators. These assignments of energy and spin quantum numbers have been verified in [\#] by explicitly solving the linearized equations motion in the near horizon string vacua.

Notice that although after reduction to $S O(7)$, the type IIA and type IIB $S O(7)$ content (3.2) coincides and therefore leads to the same tower of $S O(8)$ states, the structure of the supermultiplets is substantially different. The spectrum of type IIA on warped $\mathrm{AdS}_{3} \times S^{7}$ can alternatively be derived form that of eleven-dimensional supergravity on $\operatorname{AdS}_{4} \times S^{7}$ [12] upon dimensional reduction. Indeed, the states displayed in table 11 recombine into a single multiplet of the $O S p(8 \mid 4, \mathbb{R})$ isometry group of the eleven-dimensional vacuum.

\section{$4 \quad$ Type I supergravity on warped $\mathrm{AdS}_{3} \times S^{7}$}

The above analysis extends straightforwardly to type I supergravity on warped $\mathrm{AdS}_{3} \times S^{7}$. The starting point is now given by the decomposition under $S O(7)$ of the ten-dimensional field content $\left(\mathbf{8}_{\mathbf{v}}+\mathbf{8}_{\mathbf{s}}\right)\left(\mathbf{8}_{\mathbf{v}}+n_{v} \cdot \mathbf{1}\right)$ (with $n_{v}$ the number of vector multiplets):

$$
R_{S O(7)}^{\mathrm{I}}=\left(2+n_{v}\right) \cdot \mathbf{1}+\left(2+n_{v}\right) \cdot \mathbf{7}+\left(2+n_{v}\right) \cdot \mathbf{8}+\mathbf{2 1}+\mathbf{2 7}+\mathbf{4 8} .
$$

Again using table 1 of [

$$
\mathcal{H}_{I}=\sum_{n=0}^{\infty}\left(\mathbf{8}_{\mathbf{v}}+n_{v} \cdot \mathbf{1}\right)\left(\mathbf{8}_{\mathbf{v}}-\mathbf{8}_{\mathbf{s}}\right)(\mathbf{n} 000)
$$

The entire spectrum is collected in table 3. The quantum numbers for states sitting in the one of the first three columns can be read off directly from those in type IIB computed above. On the other hand, the state on top of the last column in table 3 carries energy $E_{0}=\frac{n}{2}+\frac{3}{2}$ and spin $s=1$.

\section{Chiral primaries in the gauge theories}

We shall finally compare the spectra of KK primaries found above with that of primary operators in the boundary theories. The nature of the dual theory is substantially different depending on whether we consider systems of fundamental or D-strings. In the former case, states in a floor of the Kaluza Klein tower below level $N$ are associated to fundamental string states with charge $N$. The physics at finite $g_{s}$ is presumably described by deformations of the twodimensional $\mathcal{N}=(8,8)$ and $\mathcal{N}=(8,0)$ sigma models associated to ten-dimensional strings 
on flat spacetimes. In the D-string case the dual gauge theory is defined via quantization of the lowest open string modes governing the low energy dynamics of $N$ nearby D-strings. They result into effective $U(N)$ and $S O(N)$ two-dimensional gauge theories for the type II and type I D-string, respectively. We focus on these D-string systems.

Let us first consider the D-string system in type IIB. The effective $U(N)$ gauge theory describing the low energy dynamics of $N$ nearby D-strings is obtained from dimensional reduction of $\mathcal{N}=1 \mathrm{SYM}$ in $D=10$ down to two dimensions. The field content comprises (besides the gauge vector field) eight adjoint scalars $\phi^{I}$, and left and right moving fermions $S^{a}, S^{\dot{a}}$, transforming in the $\mathbf{8}_{\mathbf{v}}, \mathbf{8}_{\mathbf{s}}$, and $\mathbf{8}_{\mathbf{c}}$, respectively, of the $S O(8)$ R-symmetry group. The theory is manifestly invariant under sixteen supersymmetries. The Poincare symmetry group $I S O(1,1) \times S O(8)$ and supersymmetries match the isometries and Killing spinors of the warped $\mathrm{AdS}_{3} \times S^{7}$ background, as expected.

The analysis of primary operators follows straightforwardly from that of $\mathcal{N}=4 \mathrm{SYM}$ from which the two-dimensional gauge theory descends upon dimensional reduction. As in the four-dimensional case, chiral primaries are associated to completely symmetrized, traceless operators $\mathcal{O}_{m} \equiv \operatorname{Tr}\left(\phi^{I_{1}} \ldots \phi^{I_{m}}\right), m=2,3, \ldots$, built from scalar fields in the $\mathbf{8}_{\mathbf{v}}$ and transforming in the $(m 000)$ representation of the $S O(8)$ R-symmetry group. The missing of the $m=1$ state is due the fact that $\phi^{I}$ is an $S U(N)$ rather than a $U(N)$ matrix. The remaining primaries can be found by acting with the fermionic charges $Q^{a}, \widetilde{Q}^{\dot{a}}$ on the chiral primary. Half of these charges $Q^{a}$ realize the eight on-shell supersymmetries and span the columns of table 2. The remaining $\widetilde{Q}^{\dot{a}}$ act horizontally between the various columns. The state at the bottom right is reached by $Q^{4} \widetilde{Q}^{4} \mathcal{O}_{m}=\operatorname{Tr}\left(\mathcal{F}^{4} \phi^{I_{1}} \ldots \phi^{I_{m-4}}\right)$. The results agree with those coming from the KK harmonic analysis above.

This analysis can be easily extended to the type I case. The D-string bound state dynamics is now described by an $O(N)$ gauge theory with eight scalars $\phi^{I}$ and right moving fermions $S^{a}$ in the symmetric representation of $O(N)$, eight left moving adjoint fermions $S^{\dot{a}}$, and 32 left moving fermions $\lambda^{i}$ transforming as singlets of $S O(8)$ and bifundamentals of $O(N) \times S O(32)$. The different $O(N)$ representations of left and right moving fermions compensate for the relative sign in the action of the $\Omega$-projection on type IIB D-string fields. The surviving supersymmetry is $\mathcal{N}=(8,0)$. The extra fundamentals arise from quantization of D1-D9 open strings. Chiral primaries associated to bulk supergravity modes can be derived from those in type IIB theory via $\Omega$-projection. From the gauge theory point of view, the projection of operators in the second and fourth columns of table 2 can be easily understood since they are associated to traces involving an odd number of antisymmetric matrices. In addition, we have $n_{v}=\operatorname{dim}[S O(32)]$ extra $\mathcal{N}=(8,0)$ multiplets descending from the gauge chiral primary $\operatorname{Tr}\left(\lambda^{[i} \lambda^{j]} \phi^{I_{1}} \ldots \phi^{I_{m-1}}\right)$ in the $(m-1000)$ representation of $S O(8)$. This agrees with the supergravity spectrum in table 3 .

Acknowledgements: J.F.M. wishes to thank the organizers of the workshop "The quantum structure of spacetime and the geometric nature of fundamental interactions" in Leuven, for the organization of this nice meeting. This work is partly supported by EU contract HPRN-CT2000-00122 and HPRN-CT-2000-00131. 


\section{References}

[1] J. M. Maldacena, Adv. Theor. Math. Phys. 2 (1998) 231; Int. J. Theor. Phys. 38 (1999) 1113, hep-th/9711200.

[2] N. Itzhaki, J. M. Maldacena, J. Sonnenschein and S. Yankielowicz, Phys. Rev. D 58 (1998) 046004, hep-th/9802042.

[3] H. J. Boonstra, K. Skenderis and P. K. Townsend, JHEP 9901 (1999) 003, hep-th/9807137. K. Behrndt, E. Bergshoeff, R. Halbersma and J. P. van der Schaar, Class. Quant. Grav. 16 (1999) 3517, hep-th/9907006. T. Gherghetta and Y. Oz, Phys. Rev. D 65 (2002) 046001, hep-th/0106255.

[4] J. F. Morales and H. Samtleben, JHEP 0208 (2002) 042, hep-th/0206247.

[5] R. Dijkgraaf, E. Verlinde and H. Verlinde, Nucl. Phys. B 500 (1997), hep-th/9703030; Nucl. Phys. Proc. Suppl. 68 (1998) 28.

[6] T. Banks and L. Motl, JHEP 9712 (1997) 004, hep-th/9703218; D. A. Lowe, Phys. Lett. B 403 (1997) 243, hep-th/9704041; S. J. Rey, Nucl. Phys. B 502 (1997) 170, hepth/9704158. E. Gava, J. F. Morales, K. S. Narain and G. Thompson, Nucl. Phys. B 528 (1998) 95, hep-th/9801128.

[7] C.V. Johnson, Nucl. Phys. B 537 (1999) 109, hep-th/9804200; Nucl. Phys. B 537 (1999) 144, hep-th/9806115.

[8] E. Witten, Adv. Theor. Math. Phys. 2 (1998) 253, hep-th/9802150.

[9] D. Z. Freedman, S. D. Mathur, A. Matusis and L. Rastelli, Nucl. Phys. B 546 (1999) 96, hep-th/9804058.

[10] A. Salam and J. Strathdee, Annals Phys. 141 (1982) 316.

[11] J. de Boer, Nucl. Phys. B548 (1999) 139, hep-th/9806104; JHEP 9905 (1999) 017, hepth/9812240. E. Gava, A.B. Hammou, J.F. Morales and K.S.Narain, JHEP 0103 (2001) 035 , hep-th/0102043; hep-th/0201265.

[12] B. Biran, A. Casher, F. Englert, M. Rooman and P. Spindel, Phys. Lett. B 134 (1984) 179;

A. Casher, F. Englert, H. Nicolai and M. Rooman, Nucl. Phys. B 243 (1984) 173. 